\title{
Deterioro del intercambio gaseoso en sujetos con incremento del índice de masa corporal a una altitud de 2,240 metros sobre el nivel del mar
}

\author{
Deterioration of gas exchange in subjects with an increase in body mass index at an \\ altitude of 2,240 meters above sea level
}

\author{
Luis E. Santos-Martínez ${ }^{1,2 *}$, Leticia Gómez-López³ ${ }^{3}$ Adrián Arias-Jiménez³, and Javier Quevedo-Paredes ${ }^{3}$ \\ ${ }^{1}$ Departamento de Hipertensión Pulmonar y Corazón Derecho, Unidad Médica de Alta Especialidad, Hospital de Cardiología del Centro Médico \\ Nacional, Siglo XXI, Instituto Mexicano del Seguro Social; ${ }^{2}$ Departamento de Cuidados Intensivos Posquirúrgicos Cardiovasculares, Secretaría de \\ Salud, Instituto Nacional de Cardiología Ignacio Chávez; ${ }^{3}$ Departamento de Enseñanza, Unidad Médica de Alta Especialidad, Hospital de \\ Especialidades del Centro Médico Nacional La Raza, Instituto Mexicano del Seguro Social. Ciudad de México, México
}

\begin{abstract}
Resumen
Introducción: Las alteraciones del intercambio gaseoso se han reconocido en la obesidad mórbida; sin embargo, no se conoce su comportamiento conforme se incrementa el índice de masa corporal. Objetivo: Conocer el comportamiento del intercambio gaseoso a la altura de la Ciudad de México en el desarrollo de obesidad mórbida. Métodos: Mediante un diseño transversal analítico se estudió a sujetos pareados por género y edad de cuatro grupos diferentes de índice de masa corporal $\left(\mathrm{kg} / \mathrm{m}^{2}\right)$ : normal (18.5-24.9), sobrepeso (25-29.9), obesidad (30-39.9) y obesidad mórbida ( $\left.\geq 40\right)$. Se obtuvieron sus antecedentes patológicos y demográficos, variables de gasometría arterial y espirometría simple. Las variables se determinaron de acuerdo con las características de la muestra; las diferencias entre grupos se realizaron mediante Anova de una vía con ajuste de Bonferroni, así como la correlación de Pearson para las variables relacionadas. Una $p<0.05$ se consideró con significación estadística. Resultados: Se estudió a 560 pacientes en cuatro grupos. La edad promedio fue de $49 \pm 11$ años. La mayor frecuencia de diabetes mellitus (34.29\%), hipertensión arterial (50\%) e hiperlipidemia (36.43\%) se registró en el grupo de obesidad, y la de roncador (73.57\%) en la obesidad mórbida. Se identificaron diferencias desde el grupo normal respecto de la obesidad mórbida: $\mathrm{PaCO}_{2} 31.37 \pm 2.08$ vs. $38.14 \pm 5.10 \mathrm{mmHg} ; \mathrm{PaO}_{2} 68.28 \pm 6.06$ vs. $59.86 \pm 9.28 \mathrm{mmHg}$ y SaO $293.51 \pm 1.93$ vs. $89.71 \pm 5.37 \%$, todas con $p=0.0001$. Correlación $I M C-P_{2 C O}: 0.497$, e IMC$\mathrm{PaO}_{2}:-0.365, p=0.0001$, respectivamente. Conclusiones: $A$ la altitud de la Ciudad de México y con índice de masa corporal > $30 \mathrm{~kg} / \mathrm{m}^{2}$, las variables relacionadas con el intercambio gaseoso y espirometría simple comienzan a deteriorarse; son evidentes con IMC $>40 \mathrm{~kg} / \mathrm{m}^{2}$.
\end{abstract}

Palabras clave: Obesidad. Altitud. Circulación pulmonar. Síndrome de obesidad-hipoventilación. México.

\section{Correspondencia:}

*Luis E. Santos-Martínez

E-mail: luis.santos@ cardiologia.gob.mx
Disponible en internet: 23-02-2021

Arch Cardiol Mex. 2021;91(1):7-16

www.archivoscardiologia.com

1405-9940 / @ 2020 Instituto Nacional de Cardiología Ignacio Chávez. Publicado por Permanyer. Este es un artículo open access bajo la licencia CC BY-NC-ND (http://creativecommons.org/licenses/by-nc-nd/4.0/). 


\section{Abstract}

Introduction: Alterations of gas exchange have been recognized in morbid obesity, however, it is not known how their behavior would be as the body mass index increases. Objective: To know the behavior of gas exchange at the level of Mexico City in the development of morbid obesity. Methods: Through analytical design, subjects matched by gender and age were studied from four different groups of body mass index ( $\left.\mathrm{kg} / \mathrm{m}^{2}\right)$, normal (18.5-24.9), overweight (25-29.9), obesity (30-39.9) and morbid obesity $(\geq 40)$. Their pathological and demographic antecedents, arterial blood gas and simple spirometry variables were obtained. The variables were shown according to their sample characteristic. The differences between groups were made using one way Anova with Bonferroni adjustment, as well as Pearson's correlation for the related variables. Statistical significance was considered with $p<0.05$. Results: 560 subjects were studied in 4 groups. The average age $49 \pm 11$ years old. The highest frequency of diabetes mellitus (34.29\%), arterial hypertension (50\%) and hiperlipidemia (36.43\%) was in the obesity group, and being snoring (73.57\%) in morbid obesity. There were differences from the normal group versus. morbid obesity: $\mathrm{PaCO}_{2} 31.37 \pm 2.08$ versus. $38.14 \pm 5.10 \mathrm{mmHg} ; \mathrm{PaO}_{2} 68.28 \pm 6.06$ versus. $59.86 \pm 9.28 \mathrm{mmHg}$ and $\mathrm{SaO}_{2} 93.51$ \pm 1.93 versus. $89.71 \pm 5.37 \%$, all with $p=0.0001$. The $I M C-P a C O_{2}$ correlation: 0.497 , and $I M C-\mathrm{PaO}_{2}:-0.365, p=0.0001$ respectively. Conclusions: At the altitude of Mexico City and body mass index $>30 \mathrm{~kg} / \mathrm{m}^{2}$ the variables related to gas exchange and simple spirometry begin to deteriorate; are evident with $B M I>40 \mathrm{~kg} / \mathrm{m}^{2}$.

Key words: Obesity. Altitude. Pulmonary circulation. Obesity-hypoventilation syndrome. Mexico.

\section{Introducción}

El intercambio de gases (IG) se refiere al flujo de oxígeno de los pulmones a la circulación sanguínea y la eliminación de dióxido de carbono de la sangre a los pulmones. En la práctica clínica, la gasometría arterial es la forma más común de calcularlo a través de las variables de interés, como la presión arterial de oxígeno $\left(\mathrm{PaO}_{2}\right)$, la saturación arterial de oxígeno $\left(\mathrm{SaO}_{2}\right)$ y la presión arterial de dióxido de carbono $\left(\mathrm{PaCO}_{2}\right)$, y valora la función pulmonar como un intercambiador de gases. Otra forma, más específica y especializada, consiste en determinar la capacidad para la difusión del monóxido de carbono (DLCO) y evalúa la transferencia de oxígeno del espacio alveolar a la hemoglobina de los eritrocitos en los capilares pulmonares. Se utiliza en entidades con sospecha de compromiso alveolocapilar y se requiere un equipo de función pulmonar para calcularla ${ }^{1,2}$.

La obesidad es una enfermedad crónica metabólica de muy alta prevalencia en el mundo que afecta la calidad de vida del sujeto y la función del aparato respiratorio ${ }^{3-5}$. Puede clasificarse (Organización Mundial de la Salud) de acuerdo con índices, como el índice de masa corporal (IMC, $\left.\mathrm{kg} / \mathrm{m}^{2}\right)$, el perímetro abdominal y el índice cintura/cadera ${ }^{3}$.

En la obesidad grave (IMC $\left.>30 \mathrm{~kg} / \mathrm{m}^{2}\right)$, la grasa se deposita en la cavidad abdominal, diafragma y caja torácica; se han descrito alteraciones en los volúmenes y distensibilidad pulmonar, consumo de oxígeno y aun el IG-11. Su principal manifestación es la hipoxemia de grado diverso y su mecanismo está relacionado con la formación de cortocircuitos venoarteriales (Qs/Qt), además de alteraciones de la relación ventilación/perfusión (V/Q) por la compresión grasa de las bases pulmonares y de la elevada presión intraabdominal e intratorácica con que cursan estos sujetos ${ }^{7-9,11}$.

Las alteraciones de la función pulmonar y el IG tuvieron mayor importancia cuando se señaló el compromiso del corazón y el pulmón en la obesidad grave, lo que se refirió como el síndrome cardiorrespiratorio del obeso ${ }^{7}$, ahora conocido como síndrome de obesidad-hipoventilación $(\mathrm{SOH})$ que incluye variables clínicas obtenidas de la gasometría arterial y relacionadas con el IG (definido por IMC $\geq 30 \mathrm{~kg} / \mathrm{m}^{2}$, hipercapnia crónica diurna $\left[\mathrm{PaCO}_{2} \geq 45 \mathrm{mmHg}, \mathrm{PaO}_{2}<70 \mathrm{mmHg}\right.$, valores establecidos a nivel del mar [SNM], y relacionados con trastornos respiratorios del sueño) ${ }^{4,12,13}$.

El estudio de las alteraciones de la función respiratoria casi siempre se ha realizado con diversos diseños y objetivos, pero por lo general se utilizan dos grupos, uno de control y otro con individuos con obesidad grave; sin embargo, no se conoce lo que sucede conforme el individuo aumenta su IMC en el tiempo (evolución natural), dada la dificultad de hacer el seguimiento de personas con peso normal y que evolucionan después a obesidad mórbida.

Debido a esto se prefiere estudiar a grupos independientes de acuerdo con la categoría de IMC, o a grupos con obesidad u obesidad mórbida ya establecidas. En su investigación, D’Ávila, et al. ${ }^{14}$ comunicaron el comportamiento de los flujos espiratorios (capacidad vital forzada y volumen espiratorio forzado en el primer segundo) de la prueba de espirometría simple de acuerdo con las categorías de IMC (grupos independientes), y 
como parte del IG sólo se obtuvo la saturación parcial de oxígeno $\left(\mathrm{SpO}_{2}\right)$.

No se conoce el comportamiento de las variables de la gasometría arterial vinculadas con el IG en relación con la evolución natural de la obesidad (incremento del IMC) a la altura de la Ciudad de México. En consecuencia, en este trabajo se estudió dicho comportamiento en cuatro grupos independientes de sujetos con aumento del IMC; además, en estos mismos grupos, a través de las variables de la prueba de espirometría simple (flujos espiratorios), se trató de conocer el diagnóstico funcional pulmonar prevaleciente de la función respiratoria en cada grupo de incremento del IMC.

\section{Material y métodos}

El estudio se efectuó en el Laboratorio de Fisiología Cardiopulmonar del Departamento de Hipertensión Pulmonar y Corazón Derecho de la Unidad Médica de Alta Especialidad (UMAE), del Hospital de Cardiología del Centro Médico Nacional Siglo XXI, del Instituto Mexicano del Seguro Social (IMSS), de enero de 2012 a diciembre de 2018.

Se efectuó un diseño transversal analítico y se admitió a pacientes consecutivos pareados por edad y género que presentaran los criterios de inclusión del estudio.

Se estudió a cuatro grupos de individuos nacidos y residentes de la Ciudad de México (2,240 msnm). Se categorizaron en grupos independientes de acuerdo con el índice de masa corporal ${ }^{3,4}\left(\mathrm{IMC}, \mathrm{kg} / \mathrm{m}^{2}\right)$ en normales (18.5-24.9), sobrepeso (25-29.9), obesidad (3039.9 ) y obesidad mórbida ( $\geq 40$ ).

Se aceptó para estudio a los pacientes que no tenían antecedentes de tabaquismo, ni evidencia clínica de enfermedad pulmonar o cardíaca en al menos 12 meses antes del estudio, y con antecedentes patológicos relacionados con la obesidad, como hipertensión arterial sistémica (HAS), diabetes mellitus, trastorno respiratorio durante el sueño o sospecha de ello mediante el interrogatorio de ronquido informado por la pareja.

Para el equipo de pruebas de función respiratoria se utilizaron las pruebas de función respiratoria (espirometría simple) realizadas en el equipo MasterLab-Body Standard Versión with Diffusion Option Jaeger Company Type, número de serie 101221-175110, hecho en Alemania. El equipo se calibró diariamente, antes de la jornada de trabajo.

Se usaron jeringas desechables: las muestras sanguíneas se obtuvieron con jeringas desechables DL de $1 \mathrm{ml}$ (para insulina) y longitud de la aguja de $13 \mathrm{~mm}$, calibre 27 G (Ciudad de México). Todos los sujetos estuvieron sentados al momento de obtener la muestra.

En cuanto al equipo para el análisis de muestras sanguíneas, las gasometrías arteriales se analizaron en el equipo GEM Premier 3000 modelo 5700, Instrumentation Laboratory, Lexington, Massachusetts, EE.UU. La calibración del equipo se efectuó cada 24 horas antes de su uso.

Se empleó una balanza para determinar peso y altura: báscula BAME Mod. DGN. 2412. El peso y la altura se obtuvieron en posición de pie. Para la medición del peso, a todos los pacientes se les pidió retirarse la ropa y se les proporcionó una bata hospitalaria al momento de pesarse.

Se realizó una prueba de espirometría simple: mediante el análisis de las variables relacionadas con los flujos espiratorios [(capacidad vital forzada (CVF) y el volumen espiratorio forzado en el primer segundo $\left.\left(V F_{1}\right)\right]$ se definió el patrón espirométrico funcional indicativo de normalidad, restricción pulmonar u obstrucción pulmonar. El diagnóstico espirométrico se estableció de acuerdo con el grupo de trabajo de la American Thoracic Society / European Respiratory Society ${ }^{15,16}$ (ATS/ERS) para la estandarización de la espirometría simple.

Para graduar la gravedad de la restricción pulmonar se usó el valor de la CVF como sigue: CVF 70-79\%, ligera; CVF $60-69 \%$, moderada; CVF 50-59\%, moderada-grave; CVF $35-49 \%$, grave; < $35 \%$, muy grave. A su vez, para la gravedad de la obstrucción pulmonar se utilizó el $\mathrm{VEF}_{1}$ como sigue: $\mathrm{VEF}_{1}>80 \%$, leve; $\mathrm{VEF}_{1} 50-79 \%$, moderado; $\mathrm{VEF}_{1} 30-49 \%$, grave; $<30 \%$, muy grave.

\section{Intervención}

Una vez seleccionado al individuo, se pesó y calculó su IMC; se obtuvo el consentimiento informado y se le explicó la técnica de la punción arterial. Con posterioridad se realizó la prueba de Allen modificada ${ }^{17}$; la arteria que demostró el mejor llenado fue la elegida para la punción. En posición sedente y en situación de reposo, con previa asepsia y antisepsia de la región de la arteria radial derecha o izquierda, se obtuvo la muestra mediante punción.

Obtenida la muestra sanguínea, se mantuvo en condiciones de anaerobiosis hasta su análisis. El tiempo transcurrido entre la obtención de la muestra y el análisis no superó los 3 minutos en ninguno de los casos.

A continuación se llevó a cabo la espirometría simple con calidad $A^{15}$ en su técnica de realización. Al final se registraron sus variables demográficas y antecedentes patológicos. 
Tabla 1. Ocupación de la población estudiada de acuerdo con el índice de masa corporal

\begin{tabular}{|l|c|c|c|c|}
\hline Grupo (IMC, $\left.\mathbf{k g} / \mathbf{m}^{2}\right)$ & Normal (18.5-24.9) & Sobrepeso (25-29.9) & Obesidad (30-39.9) & Obesidad Mórbida ( $\geq$ 40) \\
\hline Artes y oficios & $28(5 \%)$ & $37(6.61 \%)$ & $39(6.96 \%)$ & $36(6.43 \%)$ \\
\hline Hogar & $31(5.54 \%)$ & $32(5.71 \%)$ & $32(5.71 \%)$ & $45(8.04 \%)$ \\
\hline Técnicos & $23(4.11 \%)$ & $30(5.36 \%)$ & $24(4.29 \%)$ & $19(3.39 \%)$ \\
\hline Profesionales & $26(4.64 \%)$ & $14(2.5 \%)$ & $17(3.03 \%)$ & $14(2.5 \%)$ \\
\hline Medicina del trabajo & $15(2.68 \%)$ & $14(2.5 \%)$ & $16(2.86 \%)$ & $11(1.96 \%)$ \\
\hline Empleados de ventas & $17(3.03 \%)$ & $13(2.32 \%)$ & $12(2.15 \%)$ & $15(2.68 \%)$ \\
\hline Total & $140(25 \%)$ & $140(25 \%)$ & $140(25 \%)$ & $140(25 \%)$ \\
\hline
\end{tabular}

Para el análisis estadístico, las variables nominales y ordinales se informaron con frecuencias y porcentajes, y las numéricas con promedios y desviaciones estándar. La diferencia entre los cuatro grupos de IMC $\left(\mathrm{kg} / \mathrm{m}^{2}\right)$ se estableció mediante el análisis de varianza de una vía para grupos independientes con ajuste de Bonferroni; se obtuvo la correlación de Pearson entre las variables relacionadas con el intercambio gaseoso, IMC y las de la espirometría simple. Se aceptó una $p<0.05$ como significativa.

La muestra requerida se calculó a partir del estudio de D'Ávila, et al $^{14}$. Este estudio categorizó a los grupos de obesidad de acuerdo con el IMC y para la variable de intercambio gaseoso se valió de la saturación arterial de oxígeno $\left(\mathrm{SaO}_{2}\right)$. Por lo tanto, se utilizó este parámetro para realizar el cálculo respectivo mediante la fórmula para la comparación de medias; para una prueba de dos colas, un valor alfa (riesgo) de 0.05 y un poder del $80 \%$. La muestra calculada fue de 64 sujetos por grupo, con un total de 256, y para efectuar el pareo por género y edad se duplicó la muestra a un valor mínimo de 512.

\section{Aspectos éticos}

Se obtuvo la autorización del estudio del Comité de Investigación y de Ética de la UMAE, Hospital de Cardiología del Centro Médico Nacional, Siglo XXI, IMSS, con número R-2013-3604-4. Se obtuvo también el consentimiento informado de cada participante.

\section{Resultados}

Se estudió a 560 sujetos agrupados de acuerdo con su IMC $\left(\mathrm{kg} / \mathrm{m}^{2}\right)$. Cada grupo se integró con 140 pacientes pareados por edad y género; cada género incluyó a 70 individuos. Los tipos de actividad de las personas se distribuyeron en diferentes áreas, relacionados las más de las veces con el hogar y las artes y oficios, como se muestra en la tabla 1. La edad promedio del grupo total fue de $49 \pm 11$ años, la misma que se mantuvo similar en cada grupo pareado por género e IMC, según se muestra en las tablas 2, 3 y 4.

Los antecedentes patológicos obtenidos en esta población fueron negativos para el grupo de pacientes con IMC normal $\left(10.5-24.9 \mathrm{~kg} / \mathrm{m}^{2}\right)$. La frecuencia hombre: mujer se muestra después de cada porcentaje entre paréntesis de todos los antecedentes patológicos de cada grupo de incremento del IMC. La diabetes mellitus tipo II se presentó en los grupos de sobrepeso 6 (4.29\%; 2:4); obesidad 48 (34.29\%; 20:28) y obesidad mórbida 42 (30\%; 20:22). La diabetes mellitus tipo II fue más frecuente en la obesidad y en la obesidad mórbida sin un claro predominio de género.

Este perfil previo de comportamiento se replicó en el antecedente de hipertensión arterial sistémica: sobrepeso 5 (3.57\%; 2:3); obesidad 70 (50\%; 35:35); y obesidad mórbida 69 (49.29\%; 32:37).

El antecedente de hiperlipidemia se obtuvo en el sobrepeso 2 (1.43\%; 1:1); obesidad 51 (36.43\%; 26:25); y obesidad mórbida 46 (32.86\%; 25:21), una distribución similar a las dos anteriores.

El antecedente de roncar en los mismos grupos respectivos tuvo las siguientes cifras: 3 (2.14\%; $1: 2)$; 87 (62.14\%; 43:44); 103 (73.57\%; 47:56). La combinación de los dos antecedentes más frecuentes (HAS y ronquido) en el grupo de IMC normal y sobrepeso fueron negativos; para la obesidad 55 (39.29\%; 28:27) y para la obesidad mórbida 52 (37.14\%; 21:31), con discreto predominio del género femenino; y de los individuos que tuvieron los cuatro antecedentes se encontraron en el grupo de obesidad 29 (20.71\%; 15:14) 
Tabla 2. Diferencias en el intercambio gaseoso y patrón de espirometría simple de acuerdo con el índice de masa corporal en ambos géneros

\begin{tabular}{|c|c|c|c|c|c|}
\hline Variables (IMC, $\mathrm{kg} / \mathrm{m}^{2}$ ) & $\begin{array}{l}\text { Normal }(18.50- \\
24.99)(n=140)\end{array}$ & $\begin{array}{c}\text { Sobrepeso } \\
(25-29.99)(n=140)\end{array}$ & $\begin{array}{c}\text { Obesidad } \\
(30-39.99)(n=140)\end{array}$ & $\begin{array}{l}\text { Obesidad mórbida } \\
(\geq 40)(n=140)\end{array}$ & $\mathbf{p}$ \\
\hline Edad & $49.85 \pm 11.78$ & $49.72 \pm 11.92$ & $49.81 \pm 11.78$ & $49.82 \pm 11.85$ & $\begin{array}{l}1 \text { vs. } 2=\text { NS } \\
1 \text { vs. } 3=N S \\
1 \text { vs. } 4=N S\end{array}$ \\
\hline IMC, kg/m² & $22.88 \pm 1.51$ & $27.38 \pm 1.35$ & $33.42 \pm 2.82$ & $46.19 \pm 6.69$ & $\begin{array}{c}1 \text { vs. } 2=0.0001 \\
1 \text { vs. } 3=0.0001 \\
1 \text { vs. } 4=0.0001\end{array}$ \\
\hline $\mathrm{pH}$ & $7.41 \pm 0.02$ & $7.42 \pm 0.02$ & $7.42 \pm 0.03$ & $7.39 \pm 0.25$ & $\begin{array}{l}1 \text { vs. } 2=N S \\
1 \text { vs. } 3=N S \\
1 \text { vs. } 4=N S\end{array}$ \\
\hline $\mathrm{PaO}_{2}, \mathrm{mmHg}$ & $68.28 \pm 6.06$ & $67.54 \pm 9.33$ & $65.53 \pm 9.27$ & $59.86 \pm 9.28$ & $\begin{array}{c}1 \text { vs. } 2=N S \\
1 \text { vs. } 3=0.046 \\
1 \text { vs. } 4=0.0001\end{array}$ \\
\hline $\mathrm{PaCO}_{2}, \mathrm{mmHg}$ & $31.37 \pm 2.08$ & $32.86 \pm 4.21$ & $34.79 \pm 4.38$ & $38.14 \pm 5.10$ & $\begin{array}{l}1 \text { vs. } 2=0.0001 \\
1 \text { vs. } 3=0.0001 \\
1 \text { vs. } 4=0.0001\end{array}$ \\
\hline $\mathrm{SaO}_{2}, \%$ & $93.51 \pm 1.93$ & $93.03 \pm 3.36$ & $92.22 \pm 4.72$ & $89.71 \pm 5.37$ & $\begin{array}{c}\text { 1vs. } 2=\mathrm{NS} \\
1 \mathrm{vs} .3=0.049 \\
1 \mathrm{vs} .4=0.0001\end{array}$ \\
\hline $\mathrm{Hb}, \mathrm{g} / \mathrm{dl}$ & $13.92 \pm 1.45$ & $13.97 \pm 1.73$ & $14.82 \pm 1.71$ & $16.24 \pm 1.83$ & $\begin{array}{c}1 \text { vs. } 2=\mathrm{NS} \\
1 \text { vs. } 3=0.0001 \\
1 \text { vs. } 4=0.0001\end{array}$ \\
\hline $\begin{array}{l}\text { Patrón diagnóstico de } \\
\text { la espirometría }\end{array}$ & $\begin{array}{c}\text { Normal } \\
140(100 \%)\end{array}$ & $\begin{array}{c}\text { Restricción } 1 \\
(0.72 \%)\end{array}$ & $\begin{array}{l}\text { Restricción } \\
9(6.43 \%)\end{array}$ & $\begin{array}{l}\text { Restricción } \\
97(69.29 \%)\end{array}$ & \\
\hline
\end{tabular}

IMC: índice de masa corporal; $\mathrm{pH}$ : potencial hidrógeno; $\mathrm{PaO}_{2}$ : presión arterial de oxígeno; $\mathrm{PaCO}_{2}$ : presión arterial de dióxido de carbono; $\mathrm{SaO}_{2}$ : $\mathrm{Saturación} \mathrm{arterial} \mathrm{de}$ oxígeno; Hb: hemoglobina.

y en el de obesidad mórbida 22 (15.71\%; 12:10), sin diferencia de género.

Las diferencias observadas en el IG de acuerdo con los grupos de índice de masa corporal se registran en la tabla 2 como grupo total y, según los géneros femenino y masculino en las tabla 3 y 4 , respectivamente.

En el IG, las diferencias en las variables se observaron a medida que aumentó el IMC; la evidencia estadística señaló al grupo de obesidad y obesidad mórbida; estos valores desde lo normal hasta la obesidad mórbida fueron: $\mathrm{Hb} 13.92 \pm 1.45$ vs. $16.24 \pm 1.83 \mathrm{~g} / \mathrm{dl}, \mathrm{p}=0.0001$; $\mathrm{PaCO}_{2} 31.37 \pm 2.08$ vs. $38.14 \pm 5.10 \mathrm{mmHg}, \mathrm{p}=0.0001$; con un $\mathrm{pH} 7.41 \pm 0.02$ vs. $7.39 \pm 0.25, \mathrm{p}=\mathrm{NS}$; además de la disminución de la $\mathrm{PaO}_{2} 68.28 \pm 6.06$ vs. $59.86 \pm$ $9.28 \mathrm{mmHg}$ y de la $\mathrm{SaO}_{2} 93.51 \pm 1.93$ vs. $89.71 \pm 5.37 \%$, $p=0.0001$. El comportamiento de los cambios en relación con el género femenino y masculino es similar al grupo total, además de que tampoco se identificaron diferencias significativas entre los géneros de cada una de las categorías de IMC.
Los perfiles diagnósticos de la función respiratoria obtenidos a través de la prueba de espirometría simple se muestran en las tablas 2, 3 y 4 . En los pacientes sanos, tanto del género masculino como del femenino, el patrón fue normal en 140 (100\%); en los sujetos con sobrepeso sólo 1 persona (1.43\%) del género femenino tuvo un patrón consistente de restricción pulmonar ligera y el resto fue normal; en el grupo de obesidad en personas masculinas, 3 de ellos (4.29\%) mostraron restricción pulmonar ligera, moderada y moderada a grave, respectivamente; en las pacientes femeninas, 6 (8.57\%), 1 con restricción ligera, 4 con moderada y 1 más con moderada-grave; los demás se catalogaron como normales. En el grupo con obesidad mórbida, el total (\%) y la frecuencia de la relación hombre: mujer se establecieron como sigue: normales 40 (28.57\%; 18:22); restricción ligera 35 (25\%; 18:17); restricción moderada 36 (25.71\%; 19:17); restricción moderada-grave 20 (14.29\%; 11:9); restricción grave 6 (4.29\%; $3: 3)$; y obstrucción ligera 3 (2.14\%; 1:2). 
Tabla 3. Diferencias en el intercambio gaseoso y patrón de espirometría simple de acuerdo con el índice de masa corporal en sujetos del género femenino

\begin{tabular}{|c|c|c|c|c|c|}
\hline $\begin{array}{l}\text { Variables } \\
\left.\text { (IMC, } \mathbf{k g} / \mathbf{m}^{2}\right)\end{array}$ & $\begin{array}{c}\text { Normal (18.50-24.99) } \\
\qquad(\mathrm{n}=70)\end{array}$ & $\begin{array}{c}\text { Sobrepeso } \\
(25-29.99)(n=70)\end{array}$ & $\begin{array}{c}\text { Obesidad (30-39.99) } \\
(n=70)\end{array}$ & $\begin{array}{c}\text { Obesidad mórbida } \\
(\geq 40)(n=70)\end{array}$ & p \\
\hline Edad & $49.81 \pm 11.84$ & $49.79 \pm 11.86$ & $49.84 \pm 11.76$ & $49.84 \pm 11.84$ & $\begin{array}{l}1 \text { vs. } 2=\text { NS } \\
1 \text { vs. } 3=\text { NS } \\
1 \text { vs. } 4=N S\end{array}$ \\
\hline IMC, kg/m² & $22.85 \pm 1.47$ & $27.48 \pm 1.32$ & $33.35 \pm 2.87$ & $46.04 \pm 7.03$ & $\begin{array}{c}1 \text { vs. } 2=0.0001 \\
1 \text { vs. } 3=0.0001 \\
1 \text { vs. } 4=0.0001\end{array}$ \\
\hline $\mathrm{pH}$ & $7.42 \pm 0.02$ & $7.42 \pm 0.02$ & $7.43 \pm 0.03$ & $7.37 \pm 0.36$ & $\begin{array}{l}1 \text { vs. } 2=\mathrm{NS} \\
1 \text { vs. } 3=\mathrm{NS} \\
1 \text { vs. } 4=\mathrm{NS}\end{array}$ \\
\hline $\mathrm{PaO}_{2}, \mathrm{mmHg}$ & $67.43 \pm 6.10$ & $67.91 \pm 9.46$ & $65.87 \pm 10.46$ & $61.71 \pm 10.14$ & $\begin{array}{c}1 \text { vs. } 2=N S \\
1 \text { vs. } 3=N S \\
1 \text { vs. } 4=0.003\end{array}$ \\
\hline $\mathrm{PaCO}_{2}, \mathrm{mmHg}$ & $31.36 \pm 2.05$ & $33.36 \pm 4.38$ & $34.31 \pm 4.46$ & $37.04 \pm 5.65$ & $\begin{array}{l}1 \text { vs. } 2=N S \\
1 \text { vs. } 3=0.0001 \\
1 \text { vs. } 4=0.0001\end{array}$ \\
\hline $\mathrm{SaO}_{2}, \%$ & $93.31 \pm 2.14$ & $93.19 \pm 3.21$ & $92 \pm 5.98$ & $90.47 \pm 5.21$ & $\begin{array}{c}1 \text { vs. } 2=N S \\
1 \text { vs. } 3=N S \\
1 \text { vs. } 4=0.001\end{array}$ \\
\hline $\mathrm{Hb}, \mathrm{g} / \mathrm{dl}$ & $13.34 \pm 1.49$ & $13.77 \pm 1.43$ & $14.73 \pm 1.87$ & $16.06 \pm 1.81$ & $\begin{array}{l}1 \text { vs. } 2=N S \\
1 \text { vs. } 3=0.0001 \\
1 \text { vs. } 4=0.0001\end{array}$ \\
\hline $\begin{array}{l}\text { Patrón diagnóstico } \\
\text { de la espirometría }\end{array}$ & $\begin{array}{c}\text { Normal } \\
140(100 \%)\end{array}$ & $\begin{array}{c}\text { Restricción } 1 \\
(0.72 \%)\end{array}$ & $\begin{array}{c}\text { Restricción } \\
3(2.14 \%)\end{array}$ & $\begin{array}{l}\text { Restricción } \\
46(32.86 \%)\end{array}$ & \\
\hline
\end{tabular}

IMC; índice de masa corporal; $\mathrm{pH}$ : potencial hidrógeno; $\mathrm{PaO}_{2}$ : presión arterial de oxígeno; $\mathrm{PaCO}_{2}$ : presión arterial de dióxido de $\mathrm{carbono} \mathrm{SaO}_{2}$ : saturación arterial de oxígeno; Hb: hemoglobina.

Las correlaciones registradas del IMC y de las variables de la gasometría arterial relacionadas con el IG fueron: relación IMC-PaCO $: 0.497, \mathrm{p}=0.0001$; relación IMC-PaO $\mathrm{P}_{2}:-0.365, \mathrm{p}=0.0001$; relación IMC-hemoglobina $(\mathrm{Hb}): 0.309, \mathrm{p}=0.0001$; relación $\mathrm{PaO}_{2}-\mathrm{PaCO}_{2}:-0.607$, $\mathrm{p}=0.0001$.

Las correlaciones del IMC y de las variables relacionadas con los flujos espiratorios de la prueba de espirometría simple fueron: relación $\mathrm{IMC}_{\mathrm{MEF}}:-0.391$, $p=0.0001$; relación IMC-CVF: $-0.489, p=0.0001$.

Las correlaciones de las variables relacionadas con los flujos espiratorios de la prueba de espirometría simple y los de la gasometría arterial vinculadas con el IG se indican a continuación: relación $\mathrm{PaCO}_{2}-\mathrm{VEF}_{1}:-0.331$, $p=0.0001$; relación $\mathrm{PaO}_{2}-\mathrm{VEF}_{1:} 0.478, p=0.0001$; relación $\mathrm{PaO}_{2}$-CVF: 0.503, $\mathrm{p}=0.0001$; relación $\mathrm{PaCO}_{2}$-CVF: $-0.346, p=0.0001$.

\section{Discusión}

Este trabajo se enfocó en el comportamiento de las variables de la gasometría arterial relacionadas con el
IG; no obstante, al incluir sus antecedentes patológicos conocidos y no intervenir en el IG, también fue posible reconocer el comportamiento de sus antecedentes patológicos de acuerdo con el IMC, así como las variables relacionadas con la prueba de espirometría simple (flujos espiratorios).

Este comportamiento no se refiere directamente al tiempo de desarrollo desde la obesidad de un individuo con IMC normal hasta la obesidad mórbida, ya que sería necesario utilizar otro tipo de diseño, se requeriría un tiempo muy largo y el tamaño de la muestra sería enorme.

Este estudio es el primero en señalar, a partir de un diseño de grupos independientes, cómo podrían ser la "evolución natural" del IG, los flujos espiratorios y los antecedentes patológicos de sujetos con IMC normal hasta la obesidad mórbida (una "evolución natural" construida con las limitaciones metodológicas ya comentadas, pero válidas para estos fines).

El protocolo presupuso que la obesidad es la enfermedad metabólica de mayor prevalencia en países de occidente y vinculada con elevada morbimortalidad por 
Tabla 4. Diferencias en el intercambio gaseoso y patrón de espirometría simple de acuerdo con el índice de masa corporal en sujetos del género masculino

\begin{tabular}{|c|c|c|c|c|c|}
\hline $\begin{array}{l}\text { Variables } \\
\left.\text { (IMC, } \mathbf{k g} / \mathbf{m}^{2}\right)\end{array}$ & $\begin{array}{l}\text { Normal (18.50-24.99) } \\
\qquad(\mathrm{n}=70)\end{array}$ & $\begin{array}{c}\text { Sobrepeso } \\
(25-29.99)(n=70)\end{array}$ & $\begin{array}{c}\text { Obesidad } \\
(30-39.99)(n=70)\end{array}$ & $\begin{array}{l}\text { Obesidad mórbida } \\
(\geq 40)(n=70)\end{array}$ & $\mathbf{p}$ \\
\hline Edad & $49.89 \pm 11.80$ & $49.66 \pm 12.07$ & $49.77 \pm 11.89$ & $49.80 \pm 11.84$ & $\begin{array}{l}1 \text { vs. } 2=N S \\
1 \text { vs. } 3=N S \\
1 \text { vs. } 4=N S\end{array}$ \\
\hline IMC, $\mathrm{kg} / \mathrm{m}^{2}$ & $22.91 \pm 1.56$ & $27.27 \pm 1.37$ & $33.61 \pm 2.79$ & $46.34 \pm 6.37$ & $\begin{array}{l}1 \text { vs. } 2=0.0001 \\
1 \text { vs. } 3=0.0001 \\
1 \text { vs. } 4=0.0001\end{array}$ \\
\hline $\mathrm{pH}$ & $7.41 \pm 0.02$ & $7.42 \pm 0.02$ & $7.43 \pm 0.03$ & $7.41 \pm 0.03$ & $\begin{array}{l}1 \text { vs. } 2=N S \\
1 \text { vs. } 3=N S \\
1 \text { vs. } 4=N S\end{array}$ \\
\hline $\mathrm{PaO}_{2}, \mathrm{mmHg}$ & $69.13 \pm 5.95$ & $67.16 \pm 9.27$ & $65.19 \pm 7.98$ & $58.01 \pm 8.02$ & $\begin{array}{c}1 \text { vs. } 2=N S \\
1 \text { vs. } 3=N S \\
1 \text { vs. } 4=0.0001\end{array}$ \\
\hline $\mathrm{PaCO}_{2}, \mathrm{mmHg}$ & $31.39 \pm 2.11$ & $34.37 \pm 4$ & $35.27 \pm 4.27$ & $38.89 \pm 4.04$ & $\begin{array}{l}1 \text { vs. } 2=0.0001 \\
1 \text { vs. } 3=0.0001 \\
1 \text { vs. } 4=0.0001\end{array}$ \\
\hline $\mathrm{SaO}_{2}, \%$ & $93.70 \pm 1.69$ & $92.87 \pm 3.52$ & $92.44 \pm 3$ & $88.95 \pm 5.45$ & $\begin{array}{c}1 \text { vs. } 2=N S \\
1 \text { vs. } 3=N S \\
1 \text { vs. } 4=0.0001\end{array}$ \\
\hline $\mathrm{Hb}, \mathrm{g} / \mathrm{dl}$ & $14.01 \pm 1.39$ & $14.16 \pm 1.98$ & $14.91 \pm 1.54$ & $16.41 \pm 1.25$ & $\begin{array}{c}1 \text { vs. } 2=N S \\
1 \text { vs. } 3=N S \\
1 \text { vs. } 4=0.0001\end{array}$ \\
\hline $\begin{array}{l}\text { Patrón diagnóstico de } \\
\text { la espirometría }\end{array}$ & Normal $140(100 \%)$ & Normal $140(100 \%)$ & Restricción 6 (4.29\%) & Restricción 51 (36.43\%) & \\
\hline
\end{tabular}

alteraciones cardiovasculares, diabetes mellitus tipo II, hiperlipidemia, algunos tipos de cáncer, alteraciones osteoarticulares, síndrome metabólico y de la función respiratoria $4,6,18$.

Se ha calculado que existen en el mundo $1,600 \mathrm{mi}-$ llones de adultos con sobrepeso (IMC $\left.\geq 25 \mathrm{~kg} / \mathrm{m}^{2}\right)$ y el $25 \%$ corresponde a obesos (IMC $\left.\geq 30 \mathrm{~kg} / \mathrm{m}^{2}\right)$. En EE.UU., entre los años 1960 y 2000, el IMC promedio se incrementó alrededor del 12\%. Del año 1986 al 2000 , la prevalencia de adultos con un IMC $\geq 40 \mathrm{~kg} / \mathrm{m}^{2}$ se cuadruplicón ${ }^{19-22}$.

México ocupa el segundo lugar en cuanto a prevalencia de obesidad en personas adultas en el mundo; en términos comparativos es 10 veces mayor que la de Japón y Corea. En la población infantil ocupa el cuarto lugar mundial, sólo superado por Grecia, EE.UU. e Italia $^{23}$. El sobrepeso y la obesidad se han erigido como un grave problema de salud, social y económico. Por estas razones, la obesidad ha tenido amplia difusión en el mundo y se han diseñado en México programas sociales con la finalidad de contrarrestar sus efectos nocivos ${ }^{18,24}$.

En este trabajo se utilizó una muestra poblacional de hombres y mujeres con IMC normal, sanos y con antecedentes de enfermedades heterogéneas conocidas relacionadas con el incremento del IMC. La edad del grupo en general osciló entre la quinta y sexta décadas de la vida. Como se señaló con anterioridad, los antecedentes patológicos inician al aparecer desde el sobrepeso (IMC $\left.>25 \mathrm{~kg} / \mathrm{m}^{2}\right)$ en menor cuantía para todos ellos, y aumentan en los grupos de obesidad y obesidad mórbida; más aún, se elevan incluso hasta tener dos a cuatro antecedentes en estos dos últimos grupos.

En el trabajo de Bouloukaki, et al. ${ }^{25}$ se estudió a 6,716 pacientes con diagnóstico de apnea del sueño, síntomas adjuntos, género y prevalencia de enfermedad cardiovascular; $66 \%$ correspondió a obesos y la edad promedio fue de 52 años. Se demostró que las mujeres fueron más propensas a mostrar enfermedad cardiovascular y a referir menos síntomas de apnea de 
sueño que la población masculina. En el trabajo de los autores, con una muestra de menor tamaño, se puede observar cierto predominio de los antecedentes patológicos en el género femenino en el grupo con obesidad.

Los antecedentes patológicos se incrementan conforme aumenta el IMC, hasta la obesidad, pero llama la atención que no son mayores que los identificados en la obesidad mórbida. Es probable que esto se deba a que con este IMC mayor la morbilidad y las admisiones hospitalarias se incrementaron ${ }^{26}$ en forma tal que ya no se ven en la práctica clínica habitual, o que estos sujetos pueden incluirse en protocolos de investigación específicos, como este mismo.

En cuanto a las variables de la gasometría arterial relacionadas con el IG, la disminución de la $\mathrm{PaO}_{2}$ y $\mathrm{SaO}_{2}$, y el incremento de la $\mathrm{PaCO}_{2}$ inician su deterioro desde el grupo de sobrepeso (IMC, $25-29.9 \mathrm{~kg} / \mathrm{m}^{2}$ ) y es muy evidente en el grupo de obesidad (IMC $30 \mathrm{~kg} / \mathrm{m}^{2}$ ), por lo que estos hallazgos apoyan los programas contra la obesidad desde sujetos con evidencia de sobrepeso ${ }^{18,24}$.

La hipoxemia que ocurre en este escenario de obesidad es funcional y, al disminuir el IMC, ésta mejo$\mathrm{ra}^{7,8,10}$. El mecanismo descrito es efecto de una anomalía $V / Q$ y un cortocircuito venoarterial pulmonar ${ }^{11}$. Es posible definir su reversibilidad y señalar que la hipoxemia se debe a la obesidad mediante la maniobra de inspiración profunda ${ }^{5}$.

A diferencia de los hallazgos de este trabajo en la $\mathrm{SaO}_{2}$, en los de D'Ávila, et al. ${ }^{14}$ los cambios observados se mostraron desde un IMC $\geq 51 \mathrm{~kg} / \mathrm{m}^{2}$ (obesidad mórbida), aunque conviene indicar que sus cambios registrados en la $\mathrm{SaO}_{2}$ fueron de $\sim 95 \%$, es decir, valores normales a nivel del mar y para la altitud de la Ciudad de México ${ }^{27,28}$. Los valores de los autores en la $\mathrm{PaO}_{2}$ y la $\mathrm{SaO}_{2}$ demostraron un franco deterioro del IG desde un IMC $>30 \mathrm{~kg} / \mathrm{m}^{2}$ (grupo de obesidad), que se observa incluso antes de alcanzar la obesidad mórbi$\mathrm{da}$, a diferencia de lo que notificaron antes D'Ávila, et al. ${ }^{14}$ y tal y como ocurre en la obesidad grave ${ }^{5,7,8,11}$, según otros especialistas, sin poder precisar que en realidad ocurre desde la obesidad (según se señala en este trabajo), debido a que sólo se estudió a grupos con obesidad mórbida (IMC $>40 \mathrm{~kg} / \mathrm{m}^{2}$ ). Esta presentación más temprana del deterioro en estas variables gasométricas parece estar relacionada no sólo con el incremento del IMC, sino también con la altitud.

Por lo regular se han extrapolado los valores del intercambio gaseoso obtenidos a nivel del mar a diversos niveles de altitud, lo cual es erróneo. La altura de la Ciudad de México es de 2,240 msnm y, como se ha señalado ya, la presión barométrica disminuye progresivamente a medida que es mayor la altura sobre el nivel del $\operatorname{mar}^{27-30}$. La presión de oxígeno en el aire inspirado $\left(\mathrm{PIO}_{2}\right)$ y la presión alveolar de oxígeno $\left(\mathrm{PAO}_{2}\right)$ son directamente proporcionales a la presión barométrica, debido a lo cual ambas y la $\mathrm{PaO}_{2}$ decrecen de modo progresivo conforme se incrementa la altitud (hipoxia hipobárica). Los valores con IMC normal muestran lo anterior y los que suceden en la obesidad y obesidad mórbida parecen depender además de la altitud del incremento del IMC.

Para ejemplificar lo inexacto que puede ser utilizar variables gasométricas relacionadas con el IG obtenidas a nivel del mar y extrapoladas a otras altitudes ( $p$. ej., la Ciudad de México), se señalan los valores establecidos para el diagnóstico del síndrome de obesidad-hipoventilación: IMC $\geq 30 \mathrm{~kg} / \mathrm{m}^{2}$, hipercapnia crónica diurna $\left(\mathrm{PaCO}_{2} \geq 45 \mathrm{mmHg}\right.$ ), $\mathrm{PaO}_{2}<70 \mathrm{mmHg}$, valores determinados a nivel del mar ${ }^{5,12,13}$, donde el valor de corte para la $\mathrm{PaO}_{2}$ es de $70 \mathrm{mmHg}$. A la altitud de la Ciudad de México (2,240 msnm) lo normal sería $\sim 67 \mathrm{mmHg}$ y algo similar ocurre para la $\mathrm{PaCO}_{2}$ y, de acuerdo con lo señalado con anterioridad, quizá el IMC corporal en lugar de $30 \mathrm{~kg} / \mathrm{m}^{2}$ debería ser $>40 \mathrm{~kg} / \mathrm{m}^{2}$ para complementar el diagnóstico. Este trabajo señala la necesidad de replantear estos criterios diagnósticos a la altitud de la capital mexicana.

Asimismo, a la altura de la Ciudad de México, ¿cuándo se indicaría oxígeno complementario en estos pacientes obesos con hipoxemia? Lo habitual, de acuerdo con las guías de práctica clínica en este país ${ }^{30}$ para la enfermedad pulmonar obstructiva crónica (EPOC), es la indicación de oxígeno domiciliario a largo plazo con una $\mathrm{PaO}_{2}<55 \mathrm{mmHg}, \circ \mathrm{SaO}_{2}<88 \%$, o bien con $\mathrm{PaO}_{2}$ de 56 a $60 \mathrm{mmHg}$ en presencia de poliglobulina (hematócrito $>55 \%$ ), corazón pulmonar o hipertensión pulmonar. El efecto del oxígeno complementario se observa en la mejoría de la supervivencia, tolerancia al ejercicio, el sueño y la capacidad intelectual, en EPOC estadio IV ${ }^{31}$.

¿Es correcto en realidad utilizar este punto de corte en este grupo de pacientes? La indicación del uso de oxígeno en la hipoxemia es segura, dado que está demostrado que modifica la supervivencia en la EPOC $^{32,33}$; en realidad, ¿cuál es el punto de corte de la $\mathrm{SaO}_{2} \circ \mathrm{PaO}_{2}$ a esta altitud? y ¿en verdad conferiría un beneficio su uso en sujetos obesos u obesos mórbidos (solo o junto con las medidas establecidas para perder peso) como el referido para EPOC, en virtud del comportamiento casi siempre funcional de la hipoxemia. 
En la obesidad, las variables relacionadas con los flujos espiratorios calculados a través de la espirometría simple pueden ser normales o sugerir un proceso restrictivo debido a la reducción del volumen espiratorio forzado en el primer segundo $\left(\mathrm{VEF}_{1}\right)$ y la capacidad vital forzada $(\mathrm{CVF})^{34,35}$. Además, la reducción de la CVF puede ser grave en individuos obesos, lo que ha demostrado que el IMC se vincula con la relación $\mathrm{VEF}_{1} /$ $\mathrm{CVF}^{36}$. Por otro lado, en la obesidad grave (IMC > $62 \mathrm{~kg} / \mathrm{m}^{2}$ ) se ha demostrado una limitación al flujo aéreo (tasa de flujo medio espiratorio) y reducción de la relación $\mathrm{VEF}_{1} / \mathrm{CVF}^{37}$.

La repercusión del incremento del IMC (obesidad) en las variables de la prueba de espirometría simple fueron indicativas de restricción pulmonar a partir de la obesidad mórbida (IMC $>40 \mathrm{~kg} / \mathrm{m}^{2}$ ). En contraste con los resultados de los autores, en el trabajo de D'Ávila, et al. ${ }^{14}$ las alteraciones de las variables espirométricas fueron evidentes a partir de un IMC $>51 \mathrm{~kg} / \mathrm{m}^{2}$. Estas diferencias parecen vincularse con la talla, ya que en general fue de $1.61 \pm 0.10$ vs. $1.65 \pm 0.08 \mathrm{~m}$ de la referencia, en el análisis de los autores, además de que la población es más pequeña que otras y los valores de referencia para los estudios de espirometría parecen diferir ${ }^{15,16}$.

Las variables de la gasometría relacionadas con el IG, espirometría simple e índice de masa corporal se encuentran con una buena correlación, como se ha observado en trabajos realizados a nivel del mar ${ }^{14}$; sin embargo, en este estudio, tal relación se registró moderada, lo que puede indicar que existen otras variables que influyen en esta correlación y no se previeron en este estudio, o que el efecto de mayor correlación entre las variables se debe al mayor IMC observado en estos sujetos al nivel del mar.

Una limitación importante de este protocolo para poder construir la evolución natural del deterioro del intercambio gaseoso a medida que aumenta el IMC es precisamente el diseño metodológico. Debido a que no es posible en un tiempo restringido obtener una muestra poblacional que incremente de modo gradual el peso y muestre el espectro del daño sistémico, y por ende del aparato respiratorio, se eligió a grupos independientes y ajustados a género y edad.

Pese a las limitaciones encontradas con este diseño, se respondió la pregunta planteada, y permitió reconocer las restricciones en los diagnósticos de $\mathrm{SOH}$ y del síndrome cardiorrespiratorio del obeso a la altitud de la Ciudad de México.

\section{Conclusiones}

En individuos con índice de masa corporal $>30 \mathrm{~kg} / \mathrm{m}^{2}$ (obesidad) y a la altura de la Ciudad de México se observó que las variables relacionadas con el intercambio gaseoso, y las referidas al diagnóstico de restricción pulmonar de la espirometría simple, comienzan a deteriorarse. En ambas, estos cambios son evidentes con IMC $>40 \mathrm{~kg} / \mathrm{m}^{2}$ (obesidad mórbida).

A partir de un IMC $>25 \mathrm{~kg} / \mathrm{m}^{2}$ (sobrepeso) ya hay antecedentes patológicos y la frecuencia de los antecedentes aumentó en el grupo de obesidad; no obstante, esta frecuencia no fue mayor en el grupo de obesidad mórbida.

\section{Financiamiento}

Ninguno de los autores declara conflictos de interés.

\section{Conflicto de intereses}

Los autores declaran no tener ningún conflicto de intereses.

\section{Responsabilidades éticas}

Protección de personas y animales. Los autores declaran que para esta investigación no se han realizado experimentos en seres humanos ni en animales.

Confidencialidad de los datos. Los autores declaran que han seguido los protocolos de su centro de trabajo sobre la publicación de datos de pacientes.

Derecho a la privacidad y consentimiento informado. Los autores declaran que en este artículo no aparecen datos de pacientes.

\section{Bibliografía}

1. Hughes JM. Assessing gas exchange. Chron Respir Dis. 2007;4(4):205-14.

2. Vázquez-García JC, Gochicoa-Rangel L, Del Río-Hidalgo RF, Cid-Juárez S Silva-Cerón M, Miguel-Reyes JL, et al. Prueba de difusión pulmonar de monóxido de carbono con técnica de una sola respiración (DL,COsb). Recomendaciones y procedimiento. Neumol Cir Torax. 2016;75(2):161-72.

3. Moreno D. Definición y clasificación de la obesidad. Rev Med Clin Condes. 2012;23(2):124-28.

4. Díaz-Domínguez E, Rosas-Peralta M, Santos-Martínez LE, Rodríguez-Almendros NA, Magaña-Serrano JA, Pérez-Rodríguez G. Síndrome obesidad hipoventilación e hipertensión pulmonar. Una asociación poco conocida en México. Rev Med Inst Mex Seguro Soc. 2018;56(1):72-83.

5. Santos-Martínez LE, Martínez-Guerra ML, Bautista E, Castillo F, Castañón-Mejía A, Pulido-Zamudio T, et al. Impacto de la maniobra de inspiración profunda en el intercambio gaseoso del sujeto con obesidad severa e hipertensión arterial pulmonar asociada a síndrome de Eisenmenger. Arch Cardiol Méx. 2008:78(3);265-72.

6. Ramos P-de Lucas JM, Rodríguez-González-Moro JM, Rubio-Socorro Y. Obesidad y función pulmonar. Arch Bronconeumol. 2004;40 (Supl 5):27-37.

7. Martínez-Guerra ML, Fernández-Bonetti BP, Lupi-Herrera E, Rotberg T, Elizalde A. Cambios respiratorios en la obesidad. Estudio de 26 casos clínicos y funcionales. Arch Inst Cardiol Méx. 1975;4:12-21 
8. Martínez-Guerra ML, Fernández-Bonetti BP, Sandoval-Zárate J, Lupi-Herrera $E$. Los efectos de la posición corporal sobre la función mecánica del pulmón en el obeso. Arch Inst Cardiol Mex. 1981;51:565-70.

9. Kutcha KF. Pathophysiologic changes of obesity. Anesthesiology Clin N Am. 2005;23:421-29.

10. Parameswaran K, Todd DC, Soth M. Altered respiratory physiology in obesity. Can Respir J. 2006;13:203-10.

11. Martínez-Guerra ML, Fernández-Bonetti BP, Sandoval-Zárate J, Lupi-Herrera E. El cortocircuito veno-arterial en la obesidad, su dependencia de volumen pulmonar. Arch Inst Cardiol Mex. 1981;51:365-70.

12. Masa JF, Pépin JL, Borel JC, Mokhlesi B, Murphy PB, Sánchez-Quiroga PB. Obesity hypoventilation síndrome. Eur Respir Rev. 2019;28(1):1-14.

13. Bacelar-de Athayde RA, Bandeira de Oliveira-Filho JR, Lorenzi-Filho G Rodrigues-Genta P. Obesity hypoventilation syndrome: a current review. J Bras Pneumol. 2018;44(6):510-18.

14. D'Ávila-Melo SA, Aragão-de Melo V, Soter de Menezes-Filho R, Fábio Almeida-Santos $F$. Effects of progressive increase in body weight on lung function in six groups of body mass index. Rev Assoc Med Bras. 2011;57(5):499-505

15. Graham BL, Steenbruggen I, Miller MR, Barjaktarevic IZ, Cooper BG Hall GL, et al. Standardization of Spirometry 2019 Update. An Officia American Thoracic Society and European Respiratory Society Technical Statement. Am J Respir Crit Care Med. 2019:200(8):e70-e88.

16. Pérez-Padilla R, Valdivia G, Muiño A, López MV, Márquez MN. Spirometric reference values in 5 large Latin American cities for subjects aged 40 years or over. Arch Bronconeumol. 2006;42(7):317-25.

17. Allen EV. Thromboangeitis obliterans: Methods of diagnosis of chronic occlusive arterial lesions distal to the wrist with illustrative cases. Am J Med Sci. 1929;178:237-44.

18. Barroso-Camiade C. La obesidad, un problema de salud pública. Esp publ. 2012;15(33):200-15.

19. Prentice A, Webb F. Obesity amidst poverty. Int J Epidemiol. 2006; 35(1):24-30.

20. McTigue K, Larson JC, Valoski A, Burke G, Kotchen J, Lewis CE, et al Mortality and cardiac and vascular outcomes in extremely obese women. JAMA. 2006;296:296(1):79-86

21. Freedman DS, Khan LK, Serdula MK, Galuska DA, Dietz WH. Trend and correlates of class 3 obesity in the United States from 1990 through 2000. JAMA. 2002;288(14): 1758-61.

22. Sturm R. Increases in clinically severe obesity in the Unites States, 19862000. Arch Intern Med. 2003;163(189):2146-8

23. Dávila-Torres J, González-Izquierdo JJ, Barrera-Cruz A. Panorama de la obesidad en México. Rev Med Inst Mex Seguro Soc. 2015;53(2):240-9.
24. Barquera-Cervera S, Campos-Nonato I, Rojas R, Rivera J. Obesidad en México: epidemiología y políticas de salud para su control y prevención. Gac Med Mex. 2010;146:397-407.

25. Bouloukaki I, Mermigkis C, Markakis M, Pataka A, Alexaki I, Ermidou C, et al. Cardiovascular effect and symptom profile of obstructive sleep apnea: Does sex matter? J Clin Sleep Med. 2019;15(12):1737-45.

26. Cox ZL, Lai P, Lewis CM, Lindenfeld J. Body mass index and all-cause readmissions following acute heart failure hospitalization. Int $\mathrm{J}$ Obes (Lond). 2019. doi: 10.1038/s41366-019-0518-6.

27. Martínez-Guerra ML, Fernández-Bonetti $P$, Balvanera-Abreu A, Correa $R$ Elizalde-Ramos A, Soni-Cassani J. Valores normales de algunas medidas de la función respiratoria en la ciudad de México. Prensa Méd Mex. 1973:38(1-2):1-5.

28. Pérez-Martínez SO, Pérez-Padilla JR. Valores gasométricos en sujetos sanos reportados en la población mexicana: revisión y análisis. Rev Inv Clin. 1992; 44(3):353-362.

29. Lizardi-García D, Pérez-Padilla JR. Las altitudes sobre el nivel del mar en México: Principales poblaciones, carreteras, vías férreas, montañas y viajes aéreos. Guía para los neumólogos y sus pacientes. Neumol Cir Torax. 1993; 52(1):7-14.

30. Vázquez-García JC, Pérez-Padilla JR. Valores gasométricos estimados para las principales poblaciones y sitios a mayor altitud en México. Rev Inst Nal Enf Resp Mex. 2000;13(1):06-13.

31. Vázquez-García JC, Hernández-Zenteno RJ, Pérez-Padilla JR, Cano-Salas MC, Fernández-Vega M, Salas-Hernández j, et al. Guía de Práctica Clínica Mexicana para el diagnóstico y tratamiento de la enfermedad pulmonar obstructiva crónica. Neumol Cir Torax. 2019;78 (supl. 1):s1-s76

32. Agrawal R, Moghtader S, Ayyala U, Bandi V, Sharafkhaneh A. Update on management of stable chronic obstructive pulmonary disease. J Thorac Dis. 2019;11(Suppl 14):S1800-S09.

33. Nocturnal oxygen therapy trial group. Continuous or nocturnal oxygen therapy in hypoxemic chronic lung disease: a clinical trial. Ann Intern Med. 1980;93:391-8.

34. Beuther DA, Sutherland ER. Obesity and pulmonary function testing. J Allergy Clin Immunol. 2005;115:1100-01.

35. Collins L, Hoberty PD, Walker JF, Fletcher EC, Peiris AN. The effect of body fat distribution on pulmonary function tests. Chest. 1995;107:1298-302.

36. Lazarus R, Sparrow D, Weiss ST. Effects of obesity and fat distribution on ventilatory function. Chest. 1997;111:891-8.

37. Biring MS, Lewis MI, Liu JT, Moshenifar Z. Pulmonary physiologic changes of morbid obesity. Am J Med Sci. 1999;318:293-7. 